\title{
Experimental studies in cytoreductive surgery and hyperthermic intraperitoneal chemotherapy: overview and future directions
}

\section{Article Info}

\section{Article Notes}

Received: October 18, 2016

Accepted: December 12, 2016

\section{*Correspondence:}

Lucas Sideris, MD FRCSC, Hôpital Maisonneuve-Rosemont, 5415 boul l'Assomption

Montréal, QC, Canada, H1T 2M4, Fax: (514) 252-1463, Tel: (514) 252-5766; E-mail: lucas.sideris@umontreal.ca

C 2016 Lucas Sideris. This article is distributed under the terms of the Creative Commons Attribution 4.0 International License.

\section{Keywords}

Experimental study

Hyperthermic intraperitoneal chemotherapy

Peritoneal surface malignancy

Animal model

Pharmacokinetics

Cytoreductive surgery

HIPEC

\section{ABSTRACT}

Background: Cytoreductive surgery combined to hyperthermic intraperitoneal chemotherapy (HIPEC) is the standard of care for selected patients with peritoneal surface malignancies. Preclinical studies, especially when performed on animal models, provide a framework for improvement of this treatment. This study aims to present an overview of a single institution's experience in this setting.

Methods/Results: Review of all preclinical studies from a single center was conducted using PubMed and author databases. Multiple experiments were conducted using pig and rat models. These studies examined the impact of different surgical techniques, such as the electrovaporization of tumor nodules and the use of different suture material, in the context of HIPEC. The pharmacokinetics of multiple intraperitoneal cytotoxic agents - namely oxaliplatin, raltitrexed, irinotecan and pemetrexed - were also studied.

Conclusion: Experimental studies help guide future directions for the treatment of peritoneal surface malignancies with hyperthermic intraperitoneal chemotherapy.

\section{Introduction}

For selected patients with peritoneal surface malignancies, cytoreductive surgery (CRS) combined to hyperthermic intraperitoneal chemotherapy (HIPEC) has become the standard of care ${ }^{1-6}$. Although this approach entails morbidity rates ranging from $20.0 \%$ to $25.6 \%{ }^{7-9}$, it has been proven to be an effective treatment with encouraging survival results ${ }^{6,10}$. Preclinical studies, especially when performed on animal models, provide a valuable framework to guide further directions in improvement of this treatment. Experimental studies on CRS and HIPEC from a single institution have been reviewed using PubMed and author databases. This review aims to summarize a single center's experience in preclinical studies on CRS and HIPEC.

\section{Studies on cytoreductive surgery}

\section{Effect of chemotherapy and heat on biomechanical properties of absorbable sutures}

The quality of tissue repairs depends in part on the mechanical properties of the suture. As absorbable sutures are frequently used in cytoreductive surgery combined with HIPEC, we studied the effect of chemotherapy and heat on their biomechanical properties. Six different 3.0 absorbable sutures (Biosyn, Dexon II, Maxon, Monocryl, PDS II, and Vicryl Plus) were tested. All suture strands were incubated for a 24 -h period in $50 \mathrm{~mL}$ of saline (control), oxaliplatin 
$(1 \mathrm{mg} / \mathrm{mL})$, and mitomycin C $(0.1 \mathrm{mg} / \mathrm{mL})$ solutions at 37 and $45^{\circ} \mathrm{C}$. Suture loops were then loaded to failure using a servohydraulic testing machine. Data for tensile breaking force (TBF) and elongation rate were then collected for all samples. Under basal condition, Maxon was the strongest of all sutures with a TBF of $59.6 \pm 4.3 \mathrm{~N}(\mathrm{p}<0.01)$, and no significant difference in TBF was observed between other sutures. Heat alone had no impact on the sutures' biomechanical parameters. Exposition to mitomycin $\mathrm{C}$ at $45^{\circ} \mathrm{C}$ did not significantly affect the sutures' basal tensile properties, with Maxon remaining the strongest suture. Of all the absorbable sutures tested, Maxon is known to have the highest initial stiffness ${ }^{11}$. When incubated in oxaliplatin at $45^{\circ} \mathrm{C}$, the six suture types had a similar TBF. In all experimental conditions, multifilament sutures had a significantly lower elongation rate than monofilament sutures, and no correlations were demonstrated between elongation rate and the TBF of sutures. Those results are consistent with previous studies showing that monofilament sutures provide higher elongation rate than braided sutures ${ }^{12,13}$. In conclusion, this study showed that exposition to heated chemotherapy did not significantly affect absorbable sutures biomechanical properties ${ }^{14}$.

\section{Electrocautery effect on intestinal vascularisation in a murine model}

Electrovaporization of tumor nodules using the balltipped cautery is commonly used in cytoreductive surgery to spare as much bowel as possible from resection. Bowel perforation is the most feared complication of electrovaporization and is thought to be tightly linked to its effect on bowel wall mirovasculature. We performed a study of the impact of electrocautery (intensity and duration) on intestinal microvasculature in a murine model. A significant decrease in bowel wall microvasculature occurred with increasing intensity (coag $30 \mathrm{~W} /$ cut 40 $\mathrm{W}$ versus cut $200 \mathrm{~W}$ for 1 second, $\mathrm{p}<0.05$ ) and duration of electrocautery injury (cut $40 \mathrm{~W} 1 / 2$ seconds versus 5 seconds, $\mathrm{p}<0.05$ ). There was a $40 \%$ perforation rate when decreased bowel wall microvasculature was $25 \%$ or more. Despite similar electrocautery injury, a significantly greater microvasculature decrease was observed in the jejunum compared to ileum $(\mathrm{p}<0.05)$. Unsurprisingly, this experiment proved that the decrease in microvasculature was greater with higher intensity and duration of electrocautery and was associated with more perforations in the experimental model. The jejunum seemed more vulnerable to electrocautery injury than the ileum ${ }^{15}$.

\section{Impact of electrocautery and hyperthermic intraperitoneal chemotherapy on intestinal microvasculature in a murine model}

We repeated the latter experiment in the context of HIPEC with oxaliplatin (OX) and mitomycin C (MMC).
HIPEC with OX $(460 \mathrm{mg} / \mathrm{m} 2)$ and MMC $(10 \mathrm{mg} / \mathrm{m} 2)$ potentiated small bowel wall electrocautery injuries. We also found that MMC $(35 \mathrm{mg} / \mathrm{m} 2)$ reduced surrounding unharmed tissue microvasculature. This may explain previous studies results showing negative effect of MMC and $\mathrm{OX}$ on anastomotic healing ${ }^{16-19}$. Finally, there was no effect of hyperthermia alone on microvasculature ${ }^{20}$. A study on intestinal anastomosis breaking strength in rats showed no difference for hyperthermia up to $44^{\circ} \mathrm{C}^{21}$. Another study showed no difference in anastomotic healing after preoperative exposure to hyperthermia or radiation alone; however, the combination of both was detrimental ${ }^{22}$. The results of the current study seem to agree with the available literature, as harmful effects on tissue microvasculature after electrocautery lesions were only seen after certain HIPEC agents.

\section{Studies on HIPEC}

\section{A study of the effect of temperature on the pharmacokinetic profile of raltitrexed administered by intraperitoneal route in the rat}

We used animal models to study the pharmacokinetics of cytotoxic drugs administered intraperitoneally. We first assessed the effect of heat on the pharmacokinetic profile of intraperitoneal (IP) raltitrexed in rats. The animals have been submitted to different doses of IP raltitrexed $(2,4$, and $8 \mathrm{mg} / \mathrm{m} 2)$ at different perfusion temperatures $(37,40$ and $43^{\circ} \mathrm{C}$ ). After 25 minutes of perfusion, peritoneal fluid, portal and systemic blood were harvested and prepared for dosage of raltitrexed. Dosage was performed in a blinded fashion by High Performance Liquid Chromatography. The dose of raltitrexed administered was a determinant of its concentration in the peritoneal perfusate, the portal vein blood and systemic blood $(\mathrm{p}<0.0002)$. A previous study on IP administration of raltitrexed in pig models showed similar results ${ }^{23}$. We noticed that the perfusate temperature had no significant effect on the drug concentration in the portal vein $(p=0.29)$ and in the systemic blood $(p=0.25)$. However, temperature increased significantly $(p<0.04)$ the intracellular absorption of raltitrexed. Because the effect of raltitrexed is proportional to its intracellular concentration, this drug is very interesting to use in the setting of HIPEC as increasing temperatures lead to increased intracellular concentration with no significant increase in systemic concentration, which may be responsible for toxicity ${ }^{24}$.

\section{Pharmacokinetics of intraperitoneal irinotecan in a pig model}

In another study using a pig model, we studied the metabolism and pharmacokinetics of irinotecan (CPT-11), as well as its free and glucuronized metabolite $\mathrm{SN}-38$, when administered intraperitoneally in normothermia. This experiment showed that CPT- 11 achieved a concentration 
at least 30 times higher in the peritoneal perfusate than in the systemic blood. Peritoneal exposure to active SN-38 began at the moment of CPT-11 peritoneal instillation. A fixed and small fraction of less than $0.1 \%$ of CPT- 11 was converted into $\mathrm{SN}-38$ (both in the peritoneal perfusate and systemic blood), underlying the importance of a sufficient initial IP dose of CPT- $11^{25}$. These results are comparable to those of a mice model ${ }^{26}$ and of a human study ${ }^{27}$.

\section{Rationale for heating oxaliplatin for the intraperitoneal treatment of peritoneal carcinomatosis: a study of the effect of heat on intraperitoneal oxaliplatin using a murine model}

Using a murine model again, we studied the effect of heat on the pharmacokinetic profile of IP oxaliplatin. The rats were submitted to 3 different doses of IP oxaliplatin (460, 920, and $1840 \mathrm{mg} / \mathrm{m} 2)$ at 3 different perfusion temperatures $\left(37,40\right.$, and $\left.43^{\circ} \mathrm{C}\right)$ during 25 minutes. At the end of perfusion, samples in different compartments (peritoneum, portal blood, and systemic blood) were harvested and the concentrations of oxaliplatin were measured by high performance liquid chromatography. As the dose of IP oxaliplatin was increased, higher concentrations were observed in every compartment. This finding is in agreement with the results obtained by others ${ }^{28}$. When the temperature of IP oxaliplatin was increased, there was an increase of its peritoneal concentration (linear regression 0.38; 95\% CI: 0.28-0.47) and a decrease of its systemic blood (linear regression $-1,02$; $95 \%$ CI: -1.45 to -0.60 ) and portal blood (linear regression -1.08 ; $95 \% \mathrm{CI}:-1.70$ to -0.47 ) concentrations. Thus, proportionally to the dose administered, IP oxaliplatin lead to high concentrations of the drug in peritoneal tissues. Furthermore, heat enhanced peritoneal tissue concentration of oxaliplatin while reducing its systemic absorption. This last effect may possibly lead to decreased systemic toxicity ${ }^{29}$.

\section{Pharmacokinetics and the effect of heat on intraperitoneal pemetrexed using a murine model}

In a similar experiment, we aimed to study the effect of pemetrexed dose and perfusion temperature on the resultant pemetrexed concentration in 3 different compartments (systemic circulation, portal circulation and peritoneal tissues). The rats were submitted to 3 different doses of IP pemetrexed $(500,1000$ and $1500 \mathrm{mg} / \mathrm{m} 2)$ combined with 3 different perfusion temperatures (37, 40 and $43^{\circ} \mathrm{C}$ ) for a total duration of 25 minutes. With an increasing dose of IP pemetrexed, higher concentrations were measured in the 3 compartments tested. This is in accordance with another animal study of IP pemetrexed administration showing a 24-fold increase in the IP concentration of pemetrexed when compared with the IV route ${ }^{30}$. In the peritoneal cells, the difference between
IP doses of 500 and $1000 \mathrm{mg} / \mathrm{m} 2$ (2.03 vs. $19.17 \mu \mathrm{g} / \mathrm{g}, \mathrm{p}$ $<0.001)$ was greater than the difference between 1000 and $1500 \mathrm{mg} / \mathrm{m} 2$ (19.17 vs. $22.80 \mu \mathrm{g} / \mathrm{g}, \mathrm{p}=0.027)$. When the perfusion temperature increased, we observed a proportional increase of pemetrexed concentration in both the portal and systemic compartments, while in the peritoneal cells, the pemetrexed concentration increased up to $40^{\circ} \mathrm{C}$, after which it plateaued. Both heat and increasing doses of IP pemetrexed increased systemic and portal concentrations of the drug and enhanced its peritoneal cell concentration. However, for temperatures above $40^{\circ} \mathrm{C}$, pemetrexed concentration reached a plateau in peritoneal cells ${ }^{31}$.

\section{Future directions}

As cytoreductive surgery combined to hyperthermic intraperitoneal chemotherapy has become the standard of care for selected patients with peritoneal surface malignancies, initiatives at improving this therapeutic approach elicit a growing interest.

Homogenization of IP agent distribution in the peritoneal cavity could be refined by improvements in technologies in the delivery of HIPEC. Moreover, the use of open versus closed abdomen techniques could also have an impact on drug distribution and pharmacokinetics.

Potentiating the effect of the IP drug could improve its cytotoxic effect. Heat, dosage, and volume and output of perfusate are all variables of interest in this setting. Furthermore, the combination of IV chemotherapeutic agents to IP drugs as well as variations in intra-abdominal pressures could potentiate local cytotoxicity.

Reducing toxicity is another appealing area for improvement of CRS and HIPEC. Optimizing the duration of perfusion, refinement in the knowledge of pharmacokinetics of the different IP agents and better understanding of the impact of the solutions used for perfusion are all endpoints aiming at improving toxicity profiles of HIPEC.

\section{Conclusion}

In this review, we highlighted a few experimental studies in the field of peritoneal surface malignancies and hyperthermic intraperitoneal chemotherapy. In the context of difficulties in performing randomized clinical trials, experimental studies can guide clinicians in establishing future study directions, at lower cost.

\section{References}

1. Verwaal V J, van Ruth S, de Bree E. Randomized trial of cytoreduction and hyperthermic intraperitoneal chemotherapy versus systemic chemotherapy and palliative surgery in patients with peritoneal carcinomatosis of colorectal cancer. J Clin Oncol. 2003; 21(20): 3737-43.

2. Mohamed F, Cecil T, Moran B. A new standard of care for the management of peritoneal surface malignancy. Curr Oncol. 2011; 18(2): e84-96. 
3. Baratti D, Kusamura S, Deraco M. Diffuse malignant peritoneal mesothelioma systematic review of clinical management and biological research. J Surg Oncol. 2011; 103(8): 822-31.

4. Yan TD, Welch L, Black D. A systematic review on the efficacy of cytoreductive surgery combined with perioperative intraperitoneal chemotherapy for diffuse malignancy peritoneal mesothelioma. Ann Oncol. 2007; 18(5): 827-34.

5. Yan TD, Deraco M, Baratti D. Cytoreductive surgery and hyperthermic intraperitoneal chemotherapy for malignant peritoneal mesothelioma: multi-institutional experience. J Clin Oncol. 2009; 27(36): 6237-42.

6. Ansari N, Chandrakumaran K, Dayal S. Cytoreductive surgery and hyperthermic intraperitoneal chemotherapy in 1000 patients with perforated appendiceal epithelial tumours. Eur J Surg Oncol. 2016; 42(7): 1035-41.

7. Baratti D, Kusamura S, Iusco D. Postoperative complications after cytoreductive surgery and hyperthermic intraperitoneal chemotherapy affect long term outcome of patients with peritoneal metastases from colorectal cancer a two center study of 101 patients. Dis Colon Rectum. 2014; 57(7): 858-68.

8. Ihemelandu C, MN Mavros, P Sugarbaker. Adverse Events Postoperatively Had No Impact on Long Term Survival of Patients Treated with Cytoreductive Surgery with Heated Intraperitoneal Chemotherapy for Appendiceal Cancer with Peritoneal Metastases. Ann Surg Oncol. 2016; 23(13): 4231-4237.

9. Simkens GA, van Oudheusden TR, Luyer MD. Serious Postoperative Complications Affect Early Recurrence After Cytoreductive Surgery and HIPEC for Colorectal Peritoneal Carcinomatosis. Ann Surg Oncol. 2015; 22(8): 2656-62.

10. Gervais MK, Dubé P, McConnell Y. Cytoreductive surgery plus hyperthermic intraperitoneal chemotherapy with oxaliplatin for peritoneal carcinomatosis arising from colorectal cancer. J Surg Oncol. 2013; 108(7): 438-43.

11. Greenberg JA, RM Clark. Advances in suture material for obstetric and gynecologic surgery. Rev Obstet Gynecol. 2009; 2(3): 146-58.

12. Tomihata K, Suzuki M, Tomita N. Handling characteristics of poly L lactide co epsilon caprolactone monofilament suture. Biomed Mater Eng. 2005; 15(5): 381-91.

13. Kim JC, Lee YK, Lim BS. Comparison of tensile and knot security properties of surgical sutures. J Mater Sci Mater Med. 2007; 18(12): 2363-9.

14. Lapointe S, Zhim F, Sidéris L. Effect of chemotherapy and heat on biomechanical properties of absorbable sutures. J Surg Res. 2016; 200(1): 59-65.

15. Tremblay JF, Sideris L, Leblond FA. Electrocautery effect on intestinal vascularisation in a murine model. Int J Hyperthermia. 2016; 32(6): 643-7.

16. Fumagalli U, Trabucchi E, Soligo M. Effects of intraperitoneal chemotherapy on anastomotic healing in the rat. J Surg Res. 1991; 50(1): 82-7.
17. Makrin V, Lev Chelouche D, Even Sapir E. Intraperitoneal heated chemotherapy affects healing of experimental colonic anastomosis: an animal study. J Surg Oncol. 2005; 89(1): 18-22.

18. Pelz JO, Doerfer J, Decker M. Hyperthermic intraperitoneal chemoperfusion (HIPEC) decrease wound strength of colonic anastomosis in a rat model. Int J Colorectal Dis. 2007; 22(8): 941-7.

19. Kanellos D, Pramateftakis MG, Mantzoros I, et al. The effects of the intraperitoneal administration of oxaliplatin and 5-FU on the healing of colonic anastomoses: an experimental study. Tech Coloproctol. 2011; 15 Suppl 1: S111-5.

20. Trepanier JS, Pramateftakis MG, Mantzoros I. Impact of electrocautery and hyperthermic intraperitoneal chemotherapy on intestinal microvasculature in a murine model. Int J Hyperthermia. 2016; 32(5): 483-7.

21. Shimizu T, Maeta M, Koga S. Influence of local hyperthermia on the healing of small intestinal anastomoses in the rat. Br J Surg. 1991; 78(1): 57-9.

22. Biert J, Seifert W, de Man B. Combined preoperative irradiation and local hyperthermia delays early healing of experimental colonic anastomoses. Arch Surg. 1996; 131(10): 1037-42.

23. Nguyen D, Emond C, Leclerc Y. Pharmacokinetics studies and toxicity profile of raltitrexed used by intraperitoneal route in normothermia in a pig model. Med Sci Monit. 2003; 9(1): BR37-42.

24. Bendavid Y, Leblond FA, Dube P. A study of the effect of temperature on the pharmacokinetic profile of raltitrexed administered by intraperitoneal route in the rat. Med Sci Monit. 2005; 11(1): BR1-5.

25. Turcotte S, Sideris L, Younan R. Pharmacokinetics of intraperitoneal irinotecan in a pig model. J Surg Oncol. 2010; 101(7): 637-42.

26. Guichard S, Chatelut E, Lochon I. Comparison of the pharmacokinetics and efficacy of irinotecan after administration by the intravenous versus intraperitoneal route in mice. Cancer Chemother Pharmacol. 1998; 42(2): 165-70.

27. Elias D, Matsuhisa T, Sideris L. Heated intra-operative intraperitoneal oxaliplatin plus irinotecan after complete resection of peritoneal carcinomatosis pharmacokinetics tissue distribution and tolerance. Ann Oncol. 2004; 15(10): 1558-65.

28. Pestieau SR, Belliveau JF, Griffin H. Pharmacokinetics of intraperitoneal oxaliplatin experimental studies. J Surg Oncol. 2001; 76(2): 106-14.

29. Piche N, Leblond FA, Sidéris L. Rationale for heating oxaliplatin for the intraperitoneal treatment of peritoneal carcinomatosis a study of the effect of heat on intraperitoneal oxaliplatin using a murine model. Ann Surg. 2011; 254(1): 138-44.

30. Pestieau SR, Stuart OA, Sugarbaker PH. Multi-targeted antifolate MTA pharmacokinetics of intraperitoneal administration in a rat model. Eur J Surg Oncol. 2000; 26(7): 696-700.

31. Badrudin D, Perrault Mercier C, Bouchard Fortier A. Pharmacokinetics and the effect of heat on intraperitoneal pemetrexed using a murine model. Surg Oncol. 2016; 25(4): 435-440 